\title{
Electronic and gap properties of Sb and Bi based halide perovskites: An $a b$-initio study
}

\author{
Athanasios Koliogiorgos, Sotirios Baskoutas and Iosif Galanakis \\ Department of Materials Science, School of Natural Sciences, University of Patras, GR-26504 Patra, Greece
}

\begin{abstract}
Halide perovskites are currently under intense investigation due to their potential applications in optoelectronics and solar cells. Among them several crystallize in low symmetry lattice structures like trigonal, hexagonal, orthorhombic and monoclinic. Employing ab-initio electronic structure calculations in conjunction with generalized gradient approximation and hybrid functionals we study a series of perovskites with the formula $\mathrm{A}_{3} \mathrm{~B}_{2} \mathrm{X}_{9}$ which have been grown experimentally. A stands for a monovalent cation like $\mathrm{Cs}, \mathrm{Rb}, \mathrm{K}$ or the organic methylammonium molecule (MA), B is $\mathrm{Sb}$ or $\mathrm{Bi}$, and $\mathrm{X}$ is a halogen. Moreover we include in our study both the effect of spin-orbit coupling in the halide perovskites and the influence of the orientation disorder of the MA cation on the energy band gaps of these compounds. Most compounds under study exhibit absorption in or close to the optical regime and thus can find application in various optoelectronic devices. Our results pave the way for further investigation on the use of these materials in technology relevant applications.
\end{abstract}

Key words: Halide perovskites, Density-functional theory, Electronic Band structure PACS: 71.20.-b, 71.20.Nr, 71.15.Mb

\section{Introduction}

The continuous growth of energy consumption and the finite amount of available fossil fuels has triggered the research on alternative energy sources and especially the so-called "renewable energy sources". Among them sun is the most promising since it is an infinite energy sources and solar cells are under intense study. The most widely used material for solar cells is silicon with a history of over 60 years [1]. But the search for new cheap, earthabundant materials, which can substitute silicon in solar cells, is at a peak in recent years [2]. Among the proposed materials, perovskites [3] consist a promising family of materials for the photovoltaic (PV) industry [4-6]. Research on perovskites for $\mathrm{PVs}$ has still to address multiple issues like efficiency (PCE), the width of the energy band gap, that needs to correspond roughly to the visible spectrum, and device stability in ambient conditions prior to their commercial use. The interest on the family of perovskites also embraces other technological important research regions like optoelectronic devices [7] and catalysis [8].

Early perovskites contained oxygen and had the general structure of $\mathrm{ABO}_{3}$. To achieve charge neutrality, $\mathrm{A}$ has to be a cation of +2 valence and $\mathrm{B}$ a cation of +4 valence of dissimilar size like in $\mathrm{CaTiO}_{3}$ [9]. Latter it was found that in the same family of compounds an increasing number of materials can be categorized [5,6]. First, one can use halogen atoms instead of oxygen, giving birth to the so-called halide perovskites [10] Charge neutrality implies now that $\mathrm{A}$ is a monovalent cation and $\mathrm{B}$ a divalent.The $\mathrm{A}$ cation does not have to be a pure element but also an organic molecule and in this case the materials 
are known as hybrid or organometallic halide perovskites $[2,6,10,11]$. The choice of the two cations and the halogen atoms offers the ability to tune the photoconductive properties [12]. Among the $\mathrm{ABX}_{3}$ hybrid halide perovskites is $\mathrm{MAPbI}_{3}$, where $\mathrm{MA}$ stands for the methylammonium cation $\mathrm{CH}_{3} \mathrm{NH}_{3}{ }^{+}$, which has attracted most of the attention due to its high charge-carrier mobility and its absorption at optical regime [2,13-20].

First-principles, also known as ab-initio, electronic structure calculations are a powerful tool to study and predict the properties of compounds and thus play a decisive role in modern Materials Science. Several first-principles calculations have been devoted to the study of $\mathrm{MAPbI}_{3}$ [21-26]. The toxicity of the lead atoms led to the search for alternative hybrid halide perovskites [27,28], and firstprinciples electronic structure calculations have been devoted to the study of $\mathrm{MABX}_{3}$ compounds where $\mathrm{B}$ a divalent cation other than $\mathrm{Pb}$ and $\mathrm{X}$ an halogen atom [29-32]. In a recent publication, we studied extensively the electronic properties of cubic $\mathrm{MABX}_{3}$ compounds, where the divalent cation $\mathrm{B}$ was $\mathrm{Ca}, \mathrm{Sr}, \mathrm{Ba}, \mathrm{Zn}$., $\mathrm{Cd}, \mathrm{Hg}$, Ge, $\mathrm{Sr}$ or $\mathrm{Pb}$ and the halogen atom $\mathrm{X}$ could be $\mathrm{F}, \mathrm{Cl}, \mathrm{Br}$ or I including also the case of mixed halide perovskites [26]. Our results suggested that there were some compounds like $\mathrm{MAGeCl}_{3}$ and $\mathrm{MAGeBr}_{3}$ which could replace $\mathrm{MAPbI}_{3}$ in photovoltaics in the future, but their experimental growth in the cubic lattice structure is yet to be demonstrated.

Searching for the other possible replacements for the iodine methylammonium lead perovskite, we extend our ab-initio study to perovskites which do not crystallize in the cubic or pseudo-cubic structure, but other low-symmetry lattices like trigonal, hexagonal, orthorhombic or monoclinic [10]. In these perovskites the stoichiometry changes and the general chemical formula is now $\mathrm{A}_{3} \mathrm{~B}_{2} \mathrm{X}_{9}$, where $\mathrm{A}$ is a $\mathrm{Cs}, \mathrm{Rb}, \mathrm{K}$ or methylammonium (MA) monovalent cation, $\mathrm{B}$ is a $\mathrm{Bi}$ or $\mathrm{Sb}$ trivalent cation, and $\mathrm{X}$ is an $\mathrm{I}, \mathrm{Br}$ or $\mathrm{Cl}$ monovalent anion. Due to the large number of possible low-symmetry structures we have included in our study compounds for which the lattice structure is known experimentally. Our results show that the value of the energy band gaps vary greatly among the studied materials and thus $\mathrm{A}_{3} \mathrm{~B}_{2} \mathrm{X}_{9}$ perovskites can be promising resource of materials for photovoltaic applications. In section 2 we present shortly the details of our calculations, since they are similar to the ones in reference [26] and the structural details of the compounds under study. In sec- tion 3 we present and discuss our results, while sections 4 and 5 are devoted to the effect of spin-orbit coupling and the hybrid perovskites, respectively. Finally in section 6 we summarize and present our conclusions.

\section{Computational method}

To perform the calculations we employed the Vienna Ab-initio Simulation Package (VASP) [33]. VASP is a powerful program for performing abinitio quantum mechanical molecular dynamics simulations, developed by the Institut fur Metaliphysik of the Universitat Wien [33].In this study, similarly to the one in reference [26], we employed the projector augmented planes (PAW) pseudopotentials in reciprocal space to perform the simulations [34]. VASP also allows for self-consistent calculations including the spin-orbit coupling (SOC).

As charge density functionals, we employed, first, the Generalized Gradient Approximation (GGA) as parameterized by Perdew, Burke and Ernzerhof for solids known as the PBEsol approximation [35,36]. We used the PBEsol results to perform also calculations with the Hunde, Scuseria and Ernzerhof hybrid functional known as HSE06 [37,38]. The latter accounts for the exchange energy in a semi-empirical way considering it to be a mixture of the usual GGA exchange and of the exact Hartree-Fock exchange energy. The formula used is :

$E_{x c}=\frac{1}{4} E_{x}^{\text {exact }}+\frac{3}{4} E_{x}^{G G A}+E_{c}^{G G A}$

where the $E_{x}^{\text {exact) }}$ is the Hartree-Fock contribution and $E_{x}^{G G A}$ and $E_{c}^{G G A}$ are the respective exchange and correlation energy contributions by the GGA approximation, i.e. the PBEsol functional. Due to its nature HSE06 tends to restore the correct values of the energy band gaps which are underestimated by usual GGA calculations as shown also in the case of cubic perovskites [26]. Thus, the HSE06 is an accurate functional that can be very useful where the calculation of the optical band gap is the main concern. A drawback of this method is that it requires cpu time orders of magnitude larger that the usual GGA functionals. As a result we were able with our available computer resources to achieve convergence using HSE06 only for the compounds with the trigonal and hexagonal structures; calculations for the orthorhombic and monoclinic structures did not converge. Convergence was even more difficult when 
Table 1

$\mathrm{A}_{3} \mathrm{~B}_{2} \mathrm{X}_{9}$ compounds with their respective crystallographic structure and the reference for the experimental data. We present also the k-grid (as symmetry lowers it becomes less dense), the energy cutoff ( $\left.E_{c u t-o f f}\right)$ in eV and the calculated energy band gap values, $E_{g}$, using both the PBEsol and HSE06 functionals; in parenthesis the $E_{c u t-o f f}$ and $E_{g}$ values taking into account also the spin-orbit coupling (SOC) effect

\begin{tabular}{llllll}
\hline $\mathrm{Compound}$ & Space group & \multicolumn{2}{c}{ k-points $E_{c u t-o f f}(\mathrm{eV}) E_{g}^{\mathrm{PBEsol}}(\mathrm{eV}) E_{g}^{\mathrm{HSE06}}(\mathrm{eV}$} \\
\hline $\mathrm{Cs}_{3} \mathrm{Sb}_{2} \mathrm{I}_{9}[44]$ & Trigonal P3m1 & $5 \times 5 \times 2$ & $300(140)$ & $1.28(1.18)$ & 1.82 \\
$\mathrm{Cs}_{3} \mathrm{Sb}_{2} \mathrm{Br}_{9}[44]$ & Trigonal P3m1 & $5 \times 5 \times 2$ & $300(150)$ & $1.40(1.31)$ & 2.07 \\
$\mathrm{Cs}_{3} \mathrm{Sb}_{2} \mathrm{Cl}_{9}[45]$ & Trigonal P321 & $5 \times 5 \times 2$ & $300(170)$ & $2.12(2.07)$ & 2.97 \\
$\mathrm{Cs}_{3} \mathrm{Bi}_{2} \mathrm{I}_{9}[46]$ & Hexagonal P63/mmc $4 \times 4 \times 2$ & $190(100)$ & $1.89(0.40)$ & $2.56(0.88)$ \\
$\mathrm{Cs}_{3} \mathrm{Bi}_{2} \mathrm{Br}_{9}[44]$ & Trigonal P3m1 & $5 \times 5 \times 2$ & $300(160)$ & $2.46(1.63)$ & 3.08 \\
$\mathrm{Cs}_{3} \mathrm{Bi}_{2} \mathrm{Cl}_{9}[45]$ & Orthorhombic Pmcn $2 \times 5 \times 4$ & $150(100)$ & $2.80(1.08)$ & - \\
$\mathrm{Rb}_{3} \mathrm{Sb}_{2} \mathrm{I}_{9}[44]$ & Monoclinic Pc & $4 \times 6 \times 2$ & $110(70)$ & $1.66(1.57)$ & - \\
$\mathrm{Rb}_{3} \mathrm{Sb}_{2} \mathrm{Br}_{9}[44]$ & Trigonal P3m1 & $5 \times 5 \times 2$ & $300(200)$ & $1.55(1.45)$ & $2.21(1.98)$ \\
$\mathrm{Rb}_{3} \mathrm{Bi}_{2} \mathrm{I}_{9}[47]$ & Monoclinic Pc & $4 \times 6 \times 2$ & $100(70)$ & $1.04(1.27)$ & - \\
$\mathrm{Rb}_{3} \mathrm{Bi}_{2} \mathrm{Br}_{9}[44]$ & Orthorhombic Pnma & $2 \times 5 \times 4$ & $150(90)$ & $2.41(0.13)$ & - \\
$\mathrm{K}_{3} \mathrm{Sb}_{2} \mathrm{I}_{9}[49]$ & Monoclinic P21/n & $4 \times 5 \times 2$ & $130(80)$ & $0.68(0.48)$ & - \\
$\mathrm{K}_{3} \mathrm{Bi}_{2} \mathrm{I}_{9}[48]$ & Monoclinic P21/n & $4 \times 5 \times 2$ & $120(80)$ & $0.68(0.64)$ & - \\
$\mathrm{MA}_{3} \mathrm{Sb}_{2} \mathrm{I}_{9}[40]$ & Hexagonal P63/mmc $5 \times 5 \times 2$ & $180(100)$ & $1.73(1.52)$ & 2.36 \\
$\mathrm{MA}_{3} \mathrm{Bi}_{2} \mathrm{I}_{9}[40]$ & Hexagonal P63/mmc $5 \times 5 \times 2$ & $180(100)$ & $2.19(0.45)$ & 2.85 \\
\hline
\end{tabular}

we included also the SOC and the combined HSE06 and SOC calculations converged only for the trigonal $\mathrm{Rb}_{3} \mathrm{Sb}_{2} \mathrm{Br}_{9}$ and hexagonal $\mathrm{Cs}_{3} \mathrm{Bi}_{2} \mathrm{I}_{9}$ compounds.

As we will also discuss later when we will present our results an important parameter in the electronic band structure calculations is the cut-off energy which is the maximum kinetic energy of the plane waves used in the calculations. The larger is this value, the most accurate are the calculations. As the symmetry of the lattice lowers going from the trigonal to the monoclinic lattice, the energy cut-off used for the calculations has to lower in order to achieve convergence. Moreover the inclusion of SOC leads to an extra lowering of the possible cut-off energy. In table 1 we present both the cut-off energy, $E_{\text {cut-off }}$ in $\mathrm{eV}$, as well as the $\mathbf{k}$-points grid in the reciprocal space used in the calculations; we have used for the latter the Monkhorst-Pack scheme [39]. Test calculations with denser grids yielded roughly the same results establishing the accuracy of our results.

In table 1 we present all the compounds which we have studied together with the lattice structure and the reference to the experimental structural details. The monovalent cation is $\mathrm{Cs}, \mathrm{Rb}$ or $\mathrm{K}$ and the trivalent cation is $\mathrm{Sb}$ or $\mathrm{Bi}$, while for the halogen $\mathrm{Cl}, \mathrm{Br}$ or I are present. We have restricted ourselves to compounds for which experimental evidence exists regarding their lattice. We have also included in our study the case of the hybrid $\mathrm{MA}_{3} \mathrm{Bi}_{2} \mathrm{I}_{9}$ and $\mathrm{MA}_{3} \mathrm{Sb}_{2} \mathrm{I}_{9}$ compounds crystallizing in the hexagonal structure similar to $\mathrm{Cs}_{3} \mathrm{Bi}_{2} \mathrm{I}_{9}$ as shown in reference [40] for $\mathrm{MA}_{3} \mathrm{Bi}_{2} \mathrm{I}_{9}$; for the Sb-based compound we assumed that it crystallizes in the same lattice as the Bi-based one. As can be seen in the table, the structures of the compounds fall into four space group families (going from higher to lower symmetry): trigonal, hexagonal, orthorhombic and monoclinic. Examples of the $\mathrm{A}_{3} \mathrm{~B}_{2} \mathrm{X}_{9}$ basic lattice structures are shown in figure 1 (a-d). As can be seen from the pictures, as we progress from higher to lower symmetries, the unit cell becomes larger and thus the increased number of atoms in the unit cell leads to a large increase in the required computer power and in the computational time.

\section{Halide perovskites}

As discussed above our aim is to study the perovskite compounds with respect to their electronic properties, and especially their band gap, in order to discern which of them would be suitable for optoelectronic applications, such as solar cells. In table 1 , we present the results produced using the PBEsol and HSE06 functionals first without taking into account SOC; the effect of the SOC will be discussed 

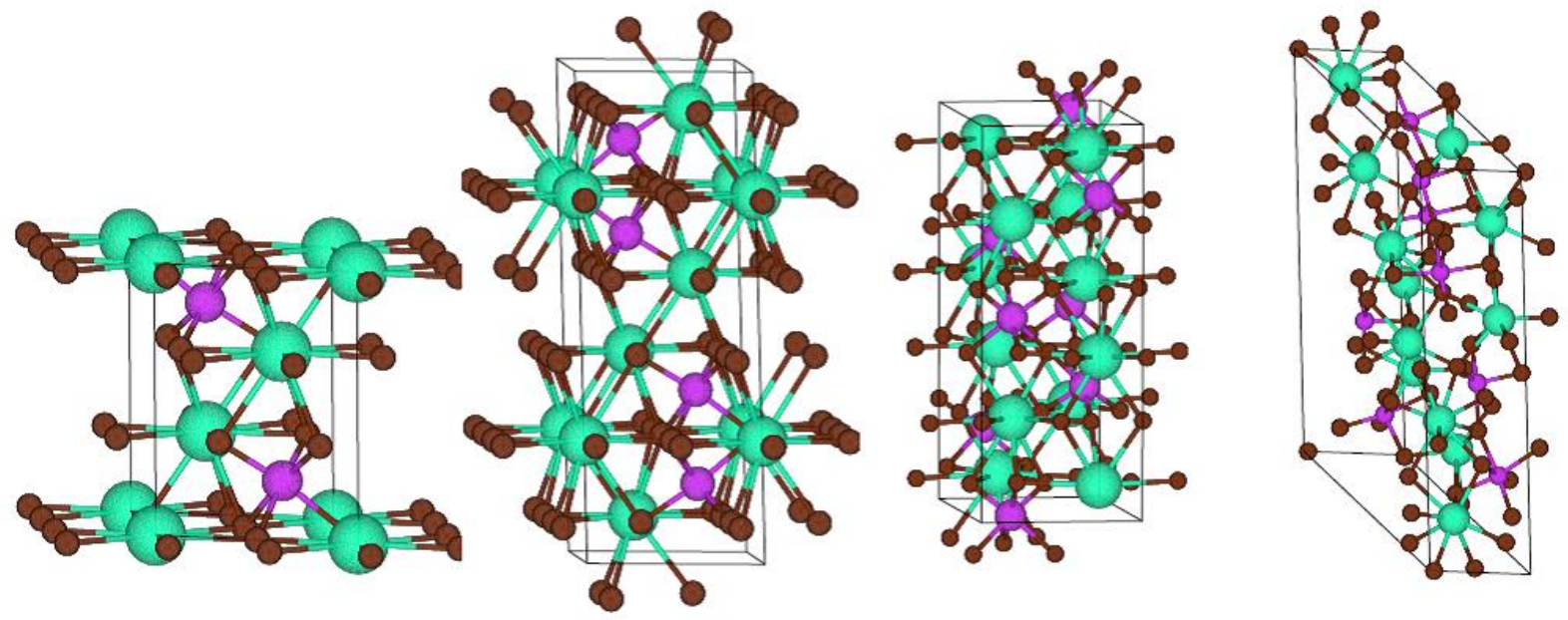

Fig. 1. Structure of $\mathrm{A}_{3} \mathrm{~B}_{2} \mathrm{X} 9$ perovskites (going from left to right): (a) trigonal structure, space group P3m1; (b) hexagonal structure, space group P63/mmc; (c) orthorhombic structure, space group Pmcn; (d) monoclinic structure, space group Pc. A atoms are in green, $\mathrm{B}$ atoms are in purple and $\mathrm{X}$ atoms in brown.

in the next section.

The first family of $\mathrm{A}_{3} \mathrm{~B}_{2} \mathrm{X}_{9}$ which we studied are the ones where $\mathrm{A}$ is Cs. For all compounds under study we have used the experimental lattice structure from the references in table 1 . The compounds where the trivalent cation is Sb crystallize all in the trigonal lattice. For this lattice structure we used a cut-off energy of $300 \mathrm{eV}$ and thus band gaps are expected to be highly accurate. Within PBEsol the width of the gap ranges between $1.28 \mathrm{eV}$ for the iodide compound up to $2.12 \mathrm{eV}$ for the chloride compound. When HSE06 was employed instead of PBEsol the values of the obtained gaps increase and reach 1.82, 2.07 and $2.97 \mathrm{eV}$ for the compounds containing as halogen atom $\mathrm{I}, \mathrm{Br}$ and $\mathrm{Cl}$, respectively. This behavior is similar to the one in cubic halide compounds studied in reference [26] and the oxygen perovskites [41]. The tendency observed with the halogen atom present in the compound can be easily explained by the density of states (DOS) presented in figure 2 for $\mathrm{Cs}_{3} \mathrm{Sb}_{2} \mathrm{I}_{9}$. The valence band is made up of the halogen $p$-states and the conduction band from the $\mathrm{Sb} p$-states. Thus the gap opens due to the $p-p$ hybridization mechanism. This ensures that the gap values are close or within the optical regime contrary to cases where the gap opens due to a $p-d$ type hybridization and the gap is much larger [26]. Secondly as we move from $\mathrm{Cl}$ to $\mathrm{Br}$ and then to I, the valence $p$-states are located higher in energy narrowing the gap. HSE06 with respect to
PBEsol shifts the conduction band slightly higher in energy opening the gap without influencing the shape of the bands.

In the Cs-based compounds when instead of $\mathrm{Sb}$ we have the heavier isovalent $\mathrm{Bi}$, the three resulting compounds have no more the same lattice structure. $\mathrm{Cs}_{3} \mathrm{Bi}_{2} \mathrm{I}_{9}$ is hexagonal, the $\mathrm{Cs}_{3} \mathrm{Bi}_{2} \mathrm{Br}_{9}$ is trigonal and $\mathrm{Cs}_{3} \mathrm{Bi}_{2} \mathrm{Cl}_{9}$ ir orthorhombic. As mentioned above for the hexagonal and orthorhombic structures we had to use a considerably smaller cut-off energy with respect to the trigonal one, which narrows the accuracy and reliability of the results obtained for these lower symmetry systems. Moreover in the case of the orthorhombic $\mathrm{Cs}_{3} \mathrm{Bi}_{2} \mathrm{Cl}_{9}$ compound we were not able to converge the HSE06 calculations. PBEsol yielded band gaps of 1.89, 2.46 and $2.80 \mathrm{eV}$ for the I-, Br- and Cl-based compounds respectively. HSE06 further increased the values of the band gap to 2.56 and $3.08 \mathrm{eV}$ for the I and $\mathrm{Br}$ compounds, respectively. These values exceed the optical regime. The origin of the gap is similar to the Sb-based compounds discussed just above.

The next step is our study is to replace Rb for Cs. Experimentally, only the compounds with $\mathrm{Br}$ and I as halogen have been grown and the lattices vary. Only $\mathrm{Rb}_{3} \mathrm{Sb}_{3} \mathrm{Br}_{9}$ is trigonal with a PBEsol band gap of $1.55 \mathrm{eV}$ and a HSE06 band gap of $2.21 \mathrm{eV}$. For the other three compounds with the lower symmetry we were not able to converge the HSE06 calculations. Finally, we have to mention that although 

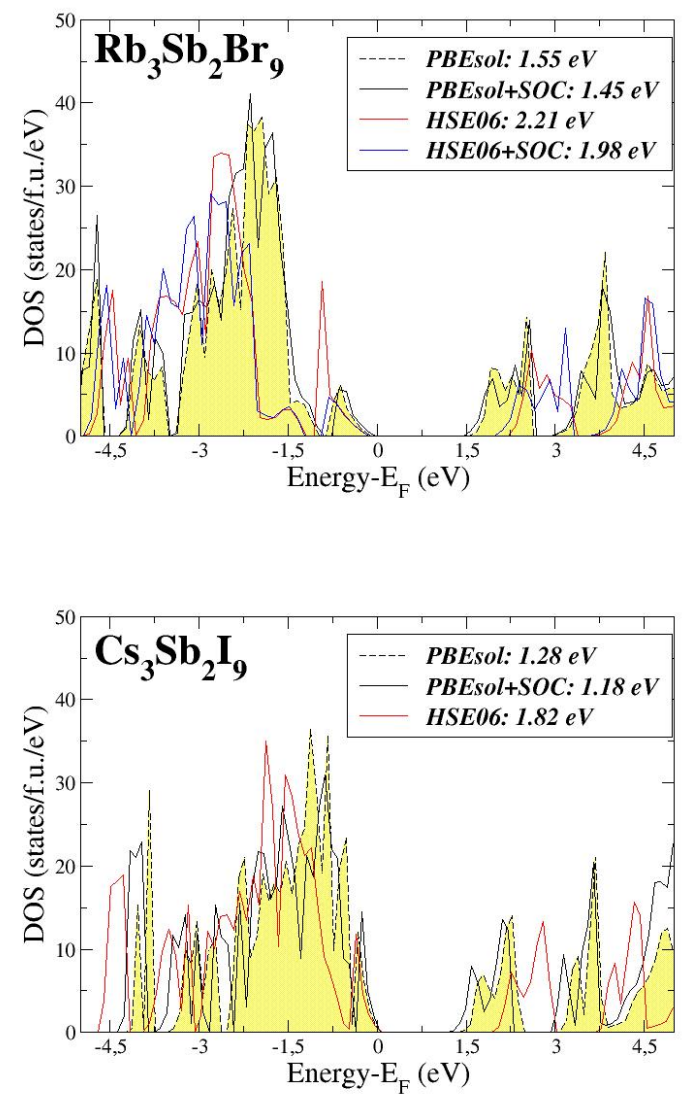

Fig. 2. Density of states for two $\mathrm{A}_{3} \mathrm{~B}_{2} \mathrm{X}_{9}$ compounds, $\mathrm{Rb}_{3} \mathrm{Sb}_{2} \mathrm{Br}_{9}$ (upper) and $\mathrm{Cs}_{3} \mathrm{Sb}_{2} \mathrm{I}_{9}$ (lower panel) using PBEsol and HSE06 functionals, and with or without SOC effect. The valence and conduction bands, as well as the band gap between them, are visible. The k-point grid and the energy cutoff are presented in table 1 . The zero energy in the horizontal axis is set to be the Fermi level.

PBEsol gives for $\mathrm{Rb}_{3} \mathrm{Bi}_{3} \mathrm{I}_{9}$ a band gap of $1.04 \mathrm{eV}$ and thus one could expect HSE06 to give a value within the optical regime, the relatively small value of the energy cut-off of $100 \mathrm{eV}$ due to its monoclinic lattice structure makes the calculated values less trustworthy than for the trigonal compounds and deviations from experiments can occur. Finally, we have also studied the $\mathrm{K}_{3} \mathrm{Sb}_{2} \mathrm{I}_{9}$ and $\mathrm{K}_{3} \mathrm{Bi}_{2} \mathrm{I} 9$ compounds. Both crystallize in the monoclinic lattice and PBEsol yields band gaps of about $0.7 \mathrm{eV}$ much smaller that the optical spectra.

For reasons of completeness we have included in table 1 also the case of the hybrid $\mathrm{MA}_{3} \mathrm{Sb}_{2} \mathrm{I}_{9}$ and $\mathrm{MA}_{3} \mathrm{Bi}_{2} \mathrm{I}_{9}$ compounds; the latter exists experimentally while for the former we have used the same hexagonal lattice structure. The cut-off energy for

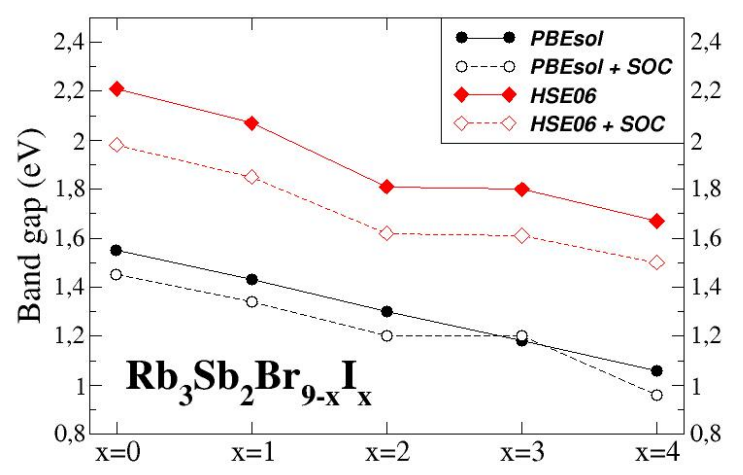

Fig. 3. Band gaps in eV of the mixed $\mathrm{Rb}_{3} \mathrm{Sb}_{2} \mathrm{Br}_{9-x} \mathrm{I}_{x}$ perovskites, with $\mathrm{x} \leq 4$. The space group for the mixed compounds is assumed to be the same as for $\mathrm{Rb}_{3} \mathrm{Sb}_{2} \mathrm{Br}_{9}$, i.e. trigonal P3m1. The energy cutoff is $300 \mathrm{eV}$ without SOC and $200 \mathrm{eV}$ with SOC. The k-point grid is $5 \times 5 \times 2$. For the HSE06+SOC case we present the expected values via extrapolation (see text for details).

our calculations is $180 \mathrm{eV}$ and we have sued a $5 \times 5 \times 2$ k-points grid in the reciprocal space. The obtained HSE06 gaps are exceeding the $2 \mathrm{eV}$ reaching a value of $2.85 \mathrm{eV}$ for the $\mathrm{MA}_{3} \mathrm{Bi}_{2} \mathrm{I}_{9}$ compound. In section 3.2 we will discuss in detail the influence of the disorder regarding the orientation of the MA cations on the obtained band gaps.

\subsection{Effect of spin-orbit coupling}

We proceeded to calculate the electronic properties of the compounds taking into account SOC using the VASP program. Previous studies suggested that spin-orbit coupling has a strong effect on the lead halide perovskites, reducing the band gap by 0.3 up to roughly $1 \mathrm{eV}$ [42], while the effect on leadfree perovskites was found to be weaker [30]. Thus it seems that the influence of SOC is materials specific. In table 1 we have included in parenthesis the energy band gap including SOC as well as the energy cut-off used in the calculations. We we able to converge the SOC calculations using PBEsol for all compounds but we achieved convergence using HSE06 only in the case of $\mathrm{Cs}_{2} \mathrm{Bi}_{2} \mathrm{I}_{9}$ and $\mathrm{Rb}_{3} \mathrm{Sb}_{2} \mathrm{Br}_{9}$ compounds. A first glimpse at the results suggests that SOC is very important and there are cases like $\mathrm{Cs}_{2} \mathrm{Bi}_{2} \mathrm{I}_{9}$ where the inclusion of SOC decreases the PBEsol band gap value by $79 \%$ and the HSE06 band gap by $66 \%$. But these value are not really trustworthy since the inclusion of SOC implies a large 
decrease of the energy cut-off used in the calculation decreasing the accuracy of the calculations.

The prototype case to study the effect of SOC is $\mathrm{Rb}_{3} \mathrm{Sb}_{2} \mathrm{Br}_{9}$ since the energy cut-off in that case is $300 \mathrm{eV}$ without SOC and $200 \mathrm{eV}$ with SOC, which should lead to accurate results in both cases. The band gap in this cases decreases by $0.1 \mathrm{eV}$ from 1.55 to $1.45 \mathrm{eV}$ in the case of PBEsol when the SOC is included, and from 2.21 to $1.98 \mathrm{eV}$ in the case of the HSE06 calculations when the SOC is included. Thus as expected SOC has a non-negligible effect on the band gap lowering it by about $10.5 \%$ in the case of the HSE06 functional. In the upper panel of figure 2 we present the DOS for all four cases under study to investigate the effect of SOC. SOC slightly moves the bands both in the case of PBEsol and HSE06 calculations without considerably affecting the shape of the initial bands without SOC.

In order to see if the decrease of the band gap is sensitive on the halogen atoms, we have partially substituted I for $\mathrm{Br}$ in $\mathrm{Rb}_{3} \mathrm{Sb}_{2} \mathrm{Br}_{9}$ resulting in the $\mathrm{Rb}_{3} \mathrm{Sb}_{2} \mathrm{Br}_{9-x} \mathrm{I}_{x}$ keeping the same trigonal structure. We have taken into account up to $x=4$ since for larger values we are closer to $\mathrm{Rb}_{3} \mathrm{Sb}_{2} \mathrm{I}_{9}$ which crystallized in the monoclinic lattice. We have performed calculations with and without SOC using the PBEsol functional and present our results in figure 3 . The calculated band gap values with SOC are about $0.1 \mathrm{eV}$ smaller than the values without SOC with the exception of $x=3$ where values are similar. Thus the effect of spin-orbit coupling does not really depend on the halogen atom but on the trivalent cation and the lattice structure. We have also made calculations using HSE06 without SOC and we present these results in figure 3 together with predicted values with SOC assuming that the SOC reduces the band gap by $10.5 \%$. The substitution of I for $\mathrm{Br}$ tunes the band gap which now falls within the optical regime and thus the $\mathrm{Rb}_{3} \mathrm{Sb}_{2} \mathrm{Br}_{9-x} \mathrm{I}_{x}$ compounds are suitable for solar cell applications.

\subsection{The case of hybrid halide perovskites}

As mentioned before, the $\mathrm{A}$ cation in the $\mathrm{A}_{3} \mathrm{~B}_{2} \mathrm{X}_{9}$ formula can be inorganic or organic. In the case of organic A, the compound is a hybrid halide perovskite. A case in point that has been studied in literature is $\mathrm{MA}_{3} \mathrm{Bi}_{2} \mathrm{I}_{9}$ perovskite where MA stands for $\mathrm{CH}_{3} \mathrm{NH}_{3}{ }^{+}$) known widely as the methylammonium cation $[40,43]$.

The crystallographic structure of $\mathrm{MA}_{3} \mathrm{Bi}_{2} \mathrm{I}_{9}$ has

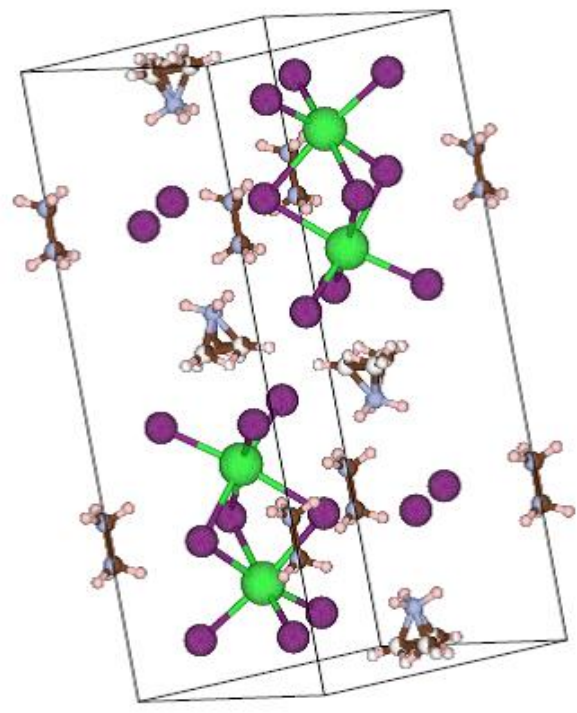

Fig. 4. $\mathrm{MA}_{3} \mathrm{Bi}_{2} \mathrm{I}_{9}$ unit cell in hexagonal structure. The atoms in the unit cell are: $\mathrm{Bi}$ in green, I in purple, $\mathrm{C}$ in brown, $\mathrm{N}$ in blue and $\mathrm{H}$ in pink. $\mathrm{CH}_{3} \mathrm{NH}_{3}{ }^{+}$cations are positioned on the edges of the unit cell and inside the unit cell, with varying orientations.

been found to be the hexagonal structure with two formula units per unit cell. Using the experimental data, we constructed the perovskite unit cell that was used in the simulation program, as seen in figure 4. As can be seen in the figure, the compound shows disorder with respect to the orientation of MA. We can distinguish two types of disorder. The two $\mathrm{CH}_{3} \mathrm{NH}_{3}$ cations on the side of the unit cell show a disorder with respect to the position of the carbon and nitrogen atoms which can exchange positions. In the case of the four inner $\mathrm{CH}_{3} \mathrm{NH}_{3}$ cations, the nitrogen atoms are fixed and the carbon atoms can have three different positions forming a fictional pyramid. Thus the question rises whether the relative positions of the $\mathrm{CH}_{3} \mathrm{NH}_{3}$ cations in the unit cell affects the width of the gap.

In order to answer the question mentioned just above we carried out electronic structure calculations using the PBEsol and assuming 12 different variations of the distribution of the orientation of the MA cations in the unit cell. Our results are summarized in table 2. For the MA cations in the inner part of the unit cell we considered 6 different possible arrangements. For the MA cations along the sides of the unit cell we considered two cases: assuming the consecutive MA cations to have either par- 
Table 2

PBEsol band gaps (in eV), in $10 \times 10 \times 4 \mathrm{k}$-point grid, for $\mathrm{MA}_{3} \mathrm{Bi}_{2} \mathrm{I}_{9}$ for different MA arrangements (see text for detals).

\begin{tabular}{lcc}
\hline Inner & \multicolumn{2}{c}{ Outer arrangements } \\
arrangement & Parallel Antiparallel \\
\hline 1 st & 1.90 & 1.75 \\
2nd & 2.18 & 2.05 \\
3rd & 1.75 & 1.75 \\
4 th & 2.04 & 2.17 \\
5 th & 1.77 & 1.64 \\
6 th & 1.89 & 1.90 \\
\hline
\end{tabular}

allel arrangement (for both the $\mathrm{C}$ atoms is located below the $\mathrm{N}$ atoms) or antiparallel arrangement. We found out that for the hybrid perovskites a larger k-points grid is needed with respect to the usual halide perovskites and thus for the calculations we have used a $10 \times 10 \times 4$ grid. This also explains the difference with the results for the same material in table 1 which corresponds to the first case presented in table 2 . As can be seen the band gaps are actually varying between the 12 studied cases ranging from $1.64 \mathrm{eV}$ to $2.18 \mathrm{eV}$. This is a large variation of more than half $\mathrm{eV}$ and may make the material suitable or not suitable for applications where absorption in a specific range of frequencies is needed. For the same inner arrangement the difference between the values for the parallel and antiparallel orientation of the outer MA cations is smallest, being at most $0.15 \mathrm{eV}$ for the 1st inner arrangement. Thus the orientation disorder of the inner MA cations should affect the obtained energy band gap in samples. We should finally note that the DOS graphs reveal that the gap is formed between the occupied $p$-states of the iodine and the empty $p$-states of Bi similar to the usual halide perovskites studied above.

\section{Summary and conclusions}

We studied using the program VASP for firstprinciple calculations the structural, electronic and optical properties of 14 halide and hybrid halide perovskites with the general formula $\mathrm{A}_{3} \mathrm{~B}_{2} \mathrm{X}_{9}$ and with trigonal, hexagonal, orthorhombic or monoclinic structure, where $\mathrm{A}$ is a monovalent cation, $\mathrm{B}$ a trivalent cation like $\mathrm{Sb}$ or $\mathrm{Bi}$ of dissimilar size and $\mathrm{X}$ a halogen. As the symmetry of the lattice lowered, the calculated band gaps were determined with smaller accuracy due to the lower energy cut- off used for the kinetic energy of the plane waves. We employed the generalized gradient approximation for all calculations as well as a more accurate hybrid functional. Where possibly, we have included also the spin-orbit coupling (SOC) in our calculations. We also studied separately two hybrid halide compounds of the group, namely $\mathrm{MA}_{3} \mathrm{Bi}_{2} \mathrm{I}_{9}$, which exists experimentally, and $\mathrm{MA}_{3} \mathrm{Sb}_{2} \mathrm{I}_{9}$ assuming the same lattice structure, were MA $\left(\mathrm{CH}_{3} \mathrm{NH}_{3}{ }^{+}\right)$is the methylammonium organic cation.

All materials under study produced band gaps ranging between 1 and $3 \mathrm{eV}$, due to the $p-p$ hybridization responsible for the appearance of the gap, and thus either are at the optical regime or near it and can find applications in optoelectronic and solar cell applications. The hybrid functional produced larger band gaps as expected for the trigonal and hexagonal materials but we could not get convergence for the lower-symmetry orthorhombic and monoclinic lattice structures. We have also carried out calculations including SOC but the low values for the energy cut-off do not allow an accurate determination of its exact effect although it leads to a significant shrinkage of the band gap. A prototype case is $\mathrm{Rb}_{3} \mathrm{Sb}_{2} \mathrm{Br}_{9}$ for which we carried out calculations combining both the hybrid functional and SOC and for which the latter led to a $10.5 \%$ decrease of the band gap. For this material we also substituted partially I for $\mathrm{Br}$ and the resulting compounds have shown a decrease in the gap with the increase of the I concentration but the effect of SOC was almost independent of the I concentration.

In the case of hybrid halide compound $\mathrm{MA}_{3} \mathrm{Bi}_{2} \mathrm{I}_{9}$, we performed calculations assuming different orientations of the MA cation. Our results suggest that the orientation of the methylammonium cations in the unit cell plays a significant role in the final band structure and band gap, and a great variation of the band gap was observed. As a result, we ascertain that, if in the synthesis process an engineering of the orientation of the organic molecules can be achieved, then a substantial tuning of the band gap is possible making the hybrid halide compounds a promising candidate for solar cell technology.

Consequently, we can conclude that it is possible to search for perovskites suitable for solar cell and optoelectronic applications among the lowsymmetry perovskites. But the width of the energy band gap is materials specific and in the case of the hybrid perovskites, the orientation of the organic molecules plays a decisive role. We expect our results to intrigue further ab-initio calculations as 
well as experiments on these promising materials.

\section{Acknowledgements}

Authors acknowledge financial support from the project PERMASOL (FFG project number: 848929).

\section{References}

[1] M.A. Green, Silicon solar cells: evolution, high-efficiency design and efficiency enhancements, Semiconduct. Sci. Technol. 8 (1993) 1-12.

[2] L. Yang, A.T. Barrows, D.G. Lidzey, T. Wang, Recent processes and challenges of organometal halide perovskite solar cells, Rep. Prog. Phys. 79 (2016) 026501.

[3] K. Hirose, R. Sinmyo, J. Hernlund, Perovskite in Earths deep interior, Science 358 (2017) 734738.

[4] J.-P. Correa-Baena, M. Saliba, T. Buonassisi, M. Grätzel, A. Abate, W. Tress, A. Hagfeldt, Promises and challenges of perovskite solar cells, Science 358 (2017) 739744 .

[5] Q. Chen, N. De Marco, Y. Yang, T. Song, C. Chen, H. Zhao, Z. Hong, H. Zhou, Y. Yang, Under the spotlight: The organic-inorganic hybrid halide perovskite for optoelectronic applications, Nano Today 10 (2015) 355396.

[6] M. Grätzel, The light and shade of perovskite solar cells, Nat. Mater.13 2014) 838-842.

[7] M.V. Kovalenko, L. Protesescu, M.I. Bodnarchuk, Properties and potential optoelectronic applications of lead halide perovskite nanocrystals, Science 358 (2017) 745750 .

[8] J. Hwang, R.R. Rao, L. Giordano, Y. Katayama, Y. Yu, Y. Shao-Horn1, Perovskites in catalysis and electrocatalysis, Science 358 (2017) 751756.

[9] M.A. Pena, J.L.G. Fierro, Chemical structure and performance of perovskite oxides, Chem. Rev. 101 (2001) 1981-2017.

[10] S.F. Hoefler, G. Trimmel, T. Rath, Progress on lead-free metal halide perovskites for photovoltaic applications: a review, Monatsh. Chem. 148 (2017) 795.

[11] G.C. Papavassiliou, G. Pagona, N. Karousis, G.A. Mousdis, I. Koutselas, A. Vassilakopoulou, Nanocrystalline/microcrystalline materials based on lead-halide units, J. Mat. Chem. 22 (2012) 8271-8280.

[12] D. Weber, Inst. Anorg. Chem. Univ. Stutt. 33B (1978) 1443-1445.

[13] M.B. Johnston, L.M. Herz, Hybrid Perovskites for Photovoltaics: Change-Carrier Recombination, Diffusion, and Radiative Efficiencies, Acc. Chem. Res. (2015). DOI: 10.1021/acs.accounts.5b00411.

[14] S. Brittman, G.W.P. Adhyaksa, E.C. Garnett, The expanding world of hybrid perovskites: materials properties and emerging applications, MRS Commun. 5 (2015) 7-26.
[15] J. M. Frost, K.T. Butler, F. Brivio, C.H. Hendon, M. van Schilfgaarde, A. Walsh, Atomistic origins of highperformance in hybrid halide perovskite solar cells, Nano Lett. 14 (2014) 2584-2590.

[16] A. Filippetti, A. Mattoni, Hybrid perovskites for photovoltaics: Insights from first principles, Phys. Rev. B 89 (2014) 125203.

[17] J. Albero, A.M. Asiri, H. Garcia, Influence of the Composition of Hybrid Perovskites on their Performance in Solar Cells, J. Mater. Chem. A (2016). DOI: 10.1039/C6TA00334F.

[18] Y. Zhao, K. Zhu, K. Organic-inorganic hybrid lead halide perovskites for optoelectronic and electronic applications, Chem. Soc. Rev. (2015). DOI: $10.1039 / \mathrm{c} 4 \mathrm{cs} 00458 \mathrm{~b}$.

[19] Y. Zhou, M. Yang, O.S. Game, W. Wu, J. Kwun, M.A. Strauss, Y. Yan, J. Huang, K. Zhu, N.P. Padture, Manipulating Crystallization of Organolead MixedHalide Thin Films in Antisolvent Baths for WideBandgap Perovskite Solar Cells, ACS Appl. Mater. Interfaces 8 (2016) 22322237.

[20] C. Quarti, E. Mosconi, J.M. Ball, V. DInnocenzo, C. Tao, S. Pathak, H.J. Snaith, A. Petrozza, F. De Angelis, Energy Environ. Sci. 9 (2016) 155.

[21] F. Brivio, A.B. Walker, A. Walsh, A. Structural and Electronic Properties of Hybrid Perovskites for High-Efficiency Thin-Film Photovoltaics from FirstPrinciples, APL Mater. 1 (2013) 042111.

[22] A. Walsh, Principles of chemical bonding and band gap engineering in hybrid organic-inorganic halide perovskites, J. Phys. Chem. C (2015). DOI: 10.1021/jp512420b.

[23] A.M.A. Leguy, J.M. Frost, A.P. McMahon, V.G. Sakai, W. Kockelmann, C. Law, X. Li, F. Foglia, A. Walsh, B.C. ORegan, J. Nelson, J.T. Cabral, P.R.F. Barnes, The dynamics of methylammonium ions in hybrid organicinorganic perovskite solar cells, Nature Commun. 6 (2015) 7124.

[24] E. Mosconi, A. Amat, M.L. Nazeeruddin, M. Grätzel, F.; De Angelis, First-principles modeling of mixed halide organometal perovskites for photovoltaic applications, J. Phys. Chem. C 117 (2013) 13902-13913.

[25] C. Motta, F. El-Mellouhi, S. Sanvito, Charge carrier mobility in hybrid halide perovskites, Nat. Sci. Reports 5 (2015) 12746.

[26] A. Koliogiorgos, S. Baskoutas, I. Galanakis, Electronic and gap properties of lead-free perfect and mixed hybrid halide perovskites: An ab-initio study, Comput. Mater. Sci. 138 (2017) 92-98.

[27] F. Hao, C.C. Stoumpos, D.H. Cao, R.P.H. Chang, M.G. Kanatzidis, Lead-free solid-state organic-inorganic halide perovskite solar cells, Nature Photonics 8 (2014) 489-494.

[28] F. Hao, C. Stoumpos, D. Cao, R. Chang, M. Kanatzidis, Lead-free solid-state organic-inorganic halide perovskite solar cells, Nat. Phot. 8 (2014) 489-494.

[29] C. Ma, M. Brik, Hybrid density-functional calculations of structural, elastic and electronic properties for a series of cubic perovskites CsMF3 $(\mathrm{M}=\mathrm{Ca}, \mathrm{Cd}, \mathrm{Hg}$ and $\mathrm{Pb})$, Comput. Mater. Sci. 58 (2012) 101-112.

[30] C. Bernal, K. Yang, First-principles hybrid functional study of the organic-inorganic 
perovskites $\mathrm{CH}_{3} \mathrm{NH}_{3} \mathrm{SnBr}_{3}$ and $\mathrm{CH}_{3} \mathrm{NH}_{3} \mathrm{SnI}_{3}$, J. Phys. Chem. C 118 (2014) 2438324388.

[31] I. Borriello, G. Cantele, D. Ninno, Ab initio investigation of hybrid organic-inorganic perovskites based on tin halides, Phys. Rev. B 77 (2008) 235214.

[32] T.J. Jacobsson, M. Pazoki, A. Hagfeldt, T. Edvinsson, Goldschmidt's rules and strontium replacement in lead halogen perovskite solar cells: Theory and preliminary experiments on $\mathrm{CH}_{3} \mathrm{NH}_{3} \mathrm{SrI}_{3}$, J. Phys. Chem. C 119 (2015) 25673-25683.

[33] G. Kresse, J. Furthmüller, Efficient iterative schemes for $a b$ initio total-energy calculations using a plane-wave basis set, Phys. Rev. B 54 (1996) 11169.

[34] J. Kresse, D. Joubert, From ultrasoft pseudopotentials to the projector augmented-wave method, Phys. Rev. B 59 (1999) 1758.

[35] J.P. Perdew, K. Burke, M. Ernzerhof, Generalized Gradient Approximation Made Simple, Phys. Rev. Lett. 77 (1998) 3865.

[36] J.P. Perdew, A. Ruzsinszky, G.I. Csonka, O.A. Vydrov, G.E. Scuseria, L.A. Constantin, X. Zhou, K. Burke, K. Restoring the Density-Gradient Expansion for Exchange in Solids and Surfaces, Phys. Rev. Lett. 100 (2008) 136406; ibid. 102 (2009) 039902(E).

[37] J. Heyd, G.E. Scuseria, M. Ernzerhof, M. Hybrid functionals based on a screened Coulomb potential, J. Chem. Phys. 118 (2003) 8207; ibid. 124 (2006) 219906(E).

[38] J. Paier, M. Marsman, K. Hummer, G. Kresse, I.C. Gerber, J.G. Angyán, Screened hybrid density functionals applied to solids, J. Chem. Phys. 124 (2006) 154709; ibid. 125 (2006) 249901E.

[39] H.J. Monkhorst, J.D. Pack, Special points for Brillouinzone integrations, Phys. Rev. B 13 (1976) 135188.

[40] K. Eckhardt, V. Bon, J. Getzschmann, J. Grothe, F. Wisser, S. Kaskel, Crystallographic insights into $\left(\mathrm{CH}_{3} \mathrm{NH}_{3}\right)_{3}\left(\mathrm{Bi}_{2} \mathrm{I}_{9}\right)$ : a new lead-free hybrid organicinorganic material as a potential absorber for photovoltaics, Chem. Commun. 52 (2016) 3058-3060.

[41] C. Franchini, Hybrid functionals applied to perovskites, J. Phys.: Condens. Matter, 26 (2016) 253202.

[42] L. Pedesseau J. Jancu, A. Rolland, E. Deleporte, C. Katan, J. Even, Electronic properties of 2D and 3D hybrid organic/inorganic perovskites for optoelectronic and photovoltaic applications, Opt. Quant. Electron 46 (2014) 1225-1232.

[43] R. Hoye, R. Brandt, A. Osherov, V. Stevanovic, S. Stranks, M. Wilson, H. Kim, A. Akey, J. Perkins, R. Kurchin, J. Poindexter, E. Wang, M. Bawendi, V. Bulovic, T. Buonassisi, Methylammonium Bismuth Iodide as a Lead-Free, Stable Hybrid Organic-Inorganic Solar Absorber, Chem. Eur. J. 22 (2016) 2605-2610.

[44] E. Peresh, $\mathrm{V}$. Sidei, O. Zubaka, I. Stercho, $\mathrm{K}_{2}\left(\mathrm{Rb}_{2}, \mathrm{Cs}_{2}, \mathrm{Tl}_{2}\right) \mathrm{TeBr}_{6}\left(\mathrm{I}_{6}\right)$ and $\mathrm{Rb}_{3}\left(\mathrm{Cs}_{3}\right) \mathrm{Sb}_{2}\left(\mathrm{Bi}_{2}\right) \mathrm{Br}_{9}\left(\mathrm{I}_{9}\right)$ perovskite compounds, Inorganic Mat. (2011) 47, 2, 208-212.

[45] C. Timmermans, S. Cholakh, G. Blasse, The luminescence of $\mathrm{Cs}_{3} \mathrm{Bi}_{2} \mathrm{Cl}_{9}$ and $\mathrm{Cs}_{3} \mathrm{Sb}_{2} \mathrm{Cl}_{9}$, J. Sol. State Chem. 46 (1983) 222-233.

[46] B. Chabot, E. Parthe, Cs3Sb2I9 and Cs3Bi2I9 with the hexagonal Cs3Cr2Cl9 structure type, Acta Cryst. B34 (1978) 645-648.
[47] V. Sidey, Y. Voroshilov, S. Kun, E. Peresh, Crystal growth and X-ray structure determination of Rb3Bi2I9, J. Alloys and Compounds 296 (2000) 53-58.

[48] A. Lehner, D. Fabini, H. Evans, C. H?bert, S. Smock, J. $\mathrm{Hu}$, H. Wang, J. Zwanziger, M. Chabinyc, R. Seshadri, Crystal and electronic structures of complex bismuth iodides $\mathrm{A}_{3} \mathrm{Bi}_{2} \mathrm{I}_{9}(\mathrm{~A}=\mathrm{K}, \mathrm{Rb}, \mathrm{Cs})$ related to perovskite: Aiding the rational design of photovoltaics, Chem. Mater. 27, 20 (2015) 7137-7148.

[49] C. Preitschaft, "Ternäre und quaternäre Materialien mit komplexen Thio-, Selenido- und Halogenid- Anionen", Dissertation, Regensburg (2004). 